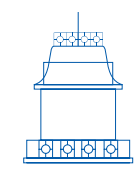

ARTÍCULOS

DE INVESTIGACIÓN

\title{
La formulación de los primeros principios en Física I 7 a la luz de la noción de generación*
}

\author{
Claudia Marisa Seggiaro \\ Universidad de Buenos Aires y Consejo Nacional de Investigaciones Científicas y Técnicas \\ (CONICET), Buenos Aires, Argentina \\ E-mail: claudiasegg@gmail.com
}

Recibido: 9 de enero de 2019 | Aceptado: 2 de julio de 2019

https://doi.org/10.17533/udea.ef.n63a02

Resumen: En el presente trabajo nos interesa analizar cómo Aristoteles formula los principios de la física a partir de la noción de generación. Nuestra tesis es que Física 7 es el "momento" de euporía que cierra un proceso que se inicia en 1-2 con la problematización acerca de la naturaleza y número de los principios. Para demostrar esto, dividiremos el trabajo en dos partes. En la primera intentaremos establecer por qué Aristóteles emprende esta investigación y cuál es el contexto argumentativo a partir del cual postula sus principios. En la segunda parte, analizaremos los supuestos con base en los cuales sostiene que materia, forma y privación son los tres principios buscados.

Palabras claves: principios, generación, materia, forma, privación

* El artículo fue realizado en el contexto de mi proyecto anual de Investigación presentado en el Consejo Nacional de Investigaciones Científicas y Técnicas, institución de la cual soy Investigadora Adjunta.

Como citar este artículo:

Seggiaro, C. M. (2021). La formulación de los primeros principios en Física 17 a la luz de la noción de generación. Estudios de Filosofía, 63, 25-46. https://doi.org/10.17533/udea.ef.n63a02

\section{OPEN A ACCESS}

Estud.filos n. ${ }^{0}$ 63. Enero-junio de 2021 | pp. 25-46 | Universidad de Antioquia | ISSN 0121-3628 | ISSN-e 2256-358X 


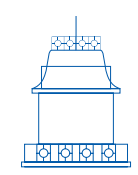

ARTÍCULOS

DE INVESTIGACIÓN

\section{The formulation of the first principles in Physics 17 in light of the notion of generation}

Abstract: In the present work we are interested in analyzing how Aristotle formulates the principles of physics from the notion of generation. Our thesis is that Physics I 7 is the "moment" of euporia that closes a process beginning in 1-2 with the problematization about the nature and number of the principles. To prove this, we will divide the work into two parts. In the first we will try to establish the argumentative context from which he postulates his principles. In the second part, we will analyze the assumptions on the basis of which it maintains that matter, form and deprivation are the three principles sought.

Keywords: principles, generation, matter, form, deprivation

Claudia Marisa Seggiaro: Doctora en Filosofía de la Universidad de Buenos Aires. Jefa de Trabajos Prácticos de Historia de la Filosofía Antigua (UBA), Investigadora Adjunta del CONICET. Principal área de trabajo: filosofía antigua.

ORCID: 0000-0001-9772-3544 


\section{Introducción}

En el presente trabajo nos interesa analizar cómo Aristóteles formula los principios de la física a partir de la noción de generación. Para ello, dividiremos el trabajo en dos partes. En la primera intentaremos establecer por qué Aristóteles emprende esta investigación y cuál es el contexto argumentativo a partir del cual postula sus principios. En la segunda parte, analizaremos los supuestos con base en los cuales sostiene que materia, forma y privación son los tres principios buscados. Nuestra tesis es que Física I 7 es el "momento" de euporía que cierra un proceso que se inicia en 1-2 con la problematización acerca de la naturaleza y número de los principios. Si bien en I 5 Aristóteles concede a sus predecesores que los contrarios deben ser principios, en 16 se percata de que debe haber un sustrato que permanezca, pues los contrarios no son capaces de devenir por sí mismos en sus opuestos. Esto genera una aparente contradicción que hace que la discusión no pueda darse por finalizada. En I 7, a partir de la noción de generación, Aristóteles intentará cancelar esta última cuestión, postulando como principios a la privación, la forma y la materia. En función de esto, nos interesa reconstruir la secuencia argumentativa por la cual en I 7 Aristóteles cree haber resuelto la problemática sobre los principios.

\section{Contexto y problemáticas en torno a la cuestión de los primeros principios}

Aristóteles comienza la Física estableciendo las condiciones de posibilidad de la ciencia. Allí establece que para que una indagación sea considerada científica debe versar sobre los primeros principios y causas (Metafísica V 1, 1013a 15-16). Esto se debe a que los principios son las causas de las cosas en dos sentidos diferentes pero complementarios. Son causa en un sentido ontológico porque son los principios constitutivos de las cosas, pero también son causa en un sentido epistemológico porque solo por su conocimiento el hombre puede saber qué son las cosas y, por lo tanto, tener un verdadero conocimiento de ellas. Sobre la base de esta prioridad ontológicoepistemológica, podemos afirmar que los principios son la condición de inteligibilidad de las cosas y, por este motivo, el verdadero objeto de la ciencia (Metafísica I, 983a 25-26. Ver Berti, 2008; Bruce \& Robertson, 2002).

Por tal motivo, tras haber establecido los requisitos para que algo sea considerado ciencia y haber sentado las bases metodológicas de su indagación, en lo que resta del primer libro de la Física, Aristóteles indagará sobre la naturaleza y número de los principios. Para ello, su análisis comenzará por el examen de las opiniones de los predecesores (Vigo, 2016, pp. 1-24). En los primeros seis capítulos se centra en las opiniones

1 Para esta cuestión véase Berti (2008) e Irwin (1988). 
de quienes sostienen alguna de estas dos tesis: 1) que solo existe un único principio o 2) que los principios son múltiples. Sin embargo, aclara que esta segunda postura es susceptible de una posterior distinción, pues, si los principios son múltiples, cabe la posibilidad de que: 2a) su número sea ilimitado o 2b) que su número sea limitado.

Cabe preguntarse por qué Aristóteles procede de este modo. ${ }^{2}$ Una posibilidad es que lo hace porque quienes sostienen esas opiniones le presentan problemas que inexorablemente tiene que tratar antes de ocuparse de la física en tanto ciencia. ${ }^{3}$ Otra alternativa es que esté construyendo adversarios con los cuales discutir y presentar los principios que posteriormente serán los puntos de partida de la física. ${ }^{4}$ Debido a que los primeros principios no pueden ser demostrados, ya sea que para discutir en torno de ellos "recree" las opiniones de sus interlocutores, o que, para legitimar la física, deba refutar a esos interlocutores, la vía que utiliza es la dialéctica. ${ }^{5}$ Los motivos por los cuales procede de este modo es que, desde su perspectiva, la dialéctica es útil a la filosofía:

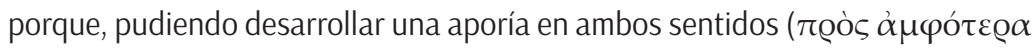
$\delta\llcorner\alpha \pi$ ọ̃ $\sigma \alpha \iota)$, discerniremos más fácilmente lo verdadero y lo falso en cada

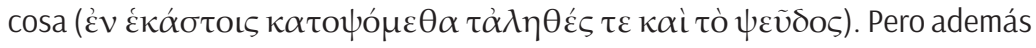

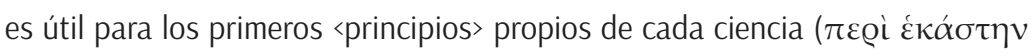

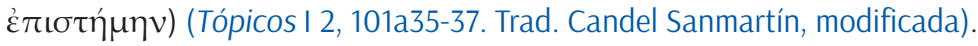

No es nuestro objetivo analizar las concepciones que Aristóteles retoma, pero en función de que forma parte de la metodología por la cual formula sus principios, haremos una mención de ellas para exponer el marco conceptual y argumentativo en el cual Aristóteles presenta sus propios principios. La primera concepción que analiza es la atribuida a los eléatas. Los eléatas ${ }^{6}$ postulan que solo hay un ser que es inmóvil y eterno. De allí, que, según Aristóteles, sostienen la existencia de un único principio. Si bien califica de erística a esta postura (Física I 2, 185a7-9), considera que discutirla tiene interés filosófico (Física I 2, 185a18-20) por los problemas que presentan. En lo que a nuestro trabajo se refiere, estas dificultades son al menos dos: 1) si solo existe un ser, este no podría ser principio, pues para que algo sea principio debe haber otra cosa respecto de la cual este sea un principio (Física I 2, 185a3-4); 2) si solo existiese

2 Para un análisis del método aristotélico de la Física, véase Couloubaritsis (1980).

3 Tal parece ser la postura que defienden Aubenque (2008, p. 354), Boeri (2006) y Rossi (2001).

4 Tal es la postura de Díaz (2014). Allí la autora afirma que Aristóteles, en rigor, está delineando "adversarios para construir un problema, como paso previo al ensayo de respuestas” (Díaz, 2014). Spangenberg, en relación con Metafísica IV (2014, pp. 152-176), también sostiene una tesis similar, que retoma de Dancy (1975) y Cassin (1989).

5 Por una cuestión de extensión, no podremos ocuparnos del rol epistemológico de la dialéctica. Para un análisis de esta cuestión véase Aguirre Santos (2015); Aubenque (2008); Bolton (1990; 2003); Brunschwig (1990) Rossi (2017), entre otros. Tal como lo indica Mié (2009): "Ias nociones que Aristóteles utiliza en Física I para analizar el cambio surgen de la discusión y el examen dialécticos de las opiniones de sus predecesores. En esto consiste un rendimiento específico de la dialéctica utilizada científicamente, lo que se conoce como método diaporemático" (p. 39).

6 Para un análisis de la lectura Aristotélica de la postura Eléata, fundamentalmente de la de Parménides, véase Cárdenas Mejía (1998). 
un ser y no pudiese existir el no ser, entonces no existiría el movimiento y, por lo tanto, la generación (Física I 7). Las consecuencias que se desprenden de esto son: 1) el conocimiento entendido como el descubrimiento de los primeros principios es imposible y 2) no es posible que existan las sustancias sensibles por estar sujetas a la generación y corrupción (Rossi, 2001). Así la física, en tanto ciencia cuyo objeto son las entidades sensibles y el movimiento al cual están sujetas, sería imposible. Para justificar la posibilidad de la física será preciso rechazar la tesis eleáta.

En Física I, 2, 184b16-19 Aristóteles atribuye también la tesis de que hay un solo principio a los "físicos", en relación con los cuales realiza una distinción ulterior (I 4, 187a12-21) entre los que suponen una materia primordial única (Tales, Anaxímenes y Heráclito), y quienes apoyan la existencia de "una multiplicidad latente en una mezcla primordial (Anaximandro)" (Echandía 1995, p. 97, nota 41). Sin embargo, en esta instancia no critica abiertamente a estos pensadores. Esto es un indicio de que, al traer a colación las concepciones de estos últimos, el objetivo de Aristóteles es diferente al que persigue cuando analiza las opiniones de los pensadores que critica (Parménides, Anaxágoras, Demócrito, etc.). Si bien Aristóteles no considerará posible una naturaleza determinada que auspicie de materia primogénita, ${ }^{7}$ es probable que retome de estas posturas la necesidad de que exista un sustrato, que debe ser principio (Bastit, 2002). Se debe destacar, por otra parte, que las posturas de algunos de estos filósofos, como por ejemplo Tales o Anaxímenes, suponen la existencia del movimiento, tesis que será central para la demostración de las principales concepciones sostenidas por Aristóteles en la Física.

La postura según la cual los principios son infinitos (2a) es retomada de Demócrito (Física I 2, 184b20-22) y de Anaxágoras (Física I 4, 187a22 ss. y I 4, 189a16-17) y es refutada al comienzo de I 6, partiendo de la base de que lo infinito es incognoscible, pues es indeterminado. Si solo hay conocimiento de lo que es determinado, y lo infinito no es determinado, entonces de lo que es infinito no puede haber ciencia (I 6, 189a12-13). Ahora bien, puesto que la ciencia es el conocimiento de los principios, estos no pueden ser infinitos, pues eso llevaría a negar la existencia de la ciencia. Por tal motivo, esta postura debe ser desechada como errónea, ya que conlleva negar el conocimiento que se pretende legitimar.

La siguiente opinión que retoma es la 2b, la de aquellos que sostienen que los principios son más de uno, pero que su número es limitado (Física I 6, 189a15), tal como lo consideraba Empédocles (Wright, 1981). Si bien Aristóteles tendrá sus reparos con respecto a la naturaleza de los principios postulados por estos pensadores, estima esta tesis acertada y, por lo tanto, la considera un buen punto de partida.

7 Como lo recalca Mié (2006): "la materia no es un esto subyacente; si lo fuera, existiría separada de los contrarios que representan forma y privación en los diferentes casos, en última instancia, sería determinable con total independencia del producto a cuya realización aporta en su calidad de sustrato material, y existiría por sí misma. Por la vía de esa errónea suposición — que desconoce la naturaleza relacional del principio material— se inauguraría la posibilidad de postular una materia primigenia, a la manera de los físicos jonios, una masa que existiría separada" (p. 82). 
Sin embargo, pese a su clasificación inicial ${ }^{8}$ Aristóteles recalca que todos los predecesores, incluso los que, como Parménides, "afirman que el todo es uno e inmóvil" (Física I 5, 188a19-20), coinciden en que los contrarios son principios (Física I 5). Esto es relevante, pues las tesis de que el número de principios es limitado y que estos son los contrarios son el punto de partida que, tras refutar las posturas 1 y $2 \mathrm{a}$, usa Aristóteles para introducir su propia concepción. Para Aristóteles:

Es necesario que los principios no provengan unos de otros ( $\mu \eta \dot{\tau} \tau \varepsilon \dot{\varepsilon} \xi \dot{\alpha} \lambda \lambda \eta \dot{\lambda} \omega \nu$ ), ni de otras cosas ( $\dot{\varepsilon} \kappa \tau o u ́ \tau \omega v)$, sino que de ellos provengan todas las cosas ( $\dot{\varepsilon} \kappa$ $\tau o u ́ \tau \omega \nu$ ). Ahora los primeros contrarios poseen estos atributos: no provienen

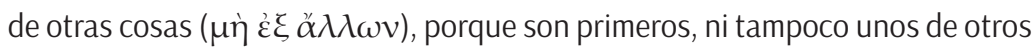

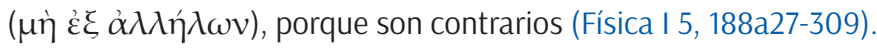

Para que algo califique como principio debe ser primero o anterior por naturaleza, pues, si necesitase de otra cosa para existir, sería ontológicamente dependiente y, por ende, no podría ser principio (Metafísica I, 983a 25-26; V 1, 1013a 16-18). Esto implica que no podrá ser a partir de otra cosa, sino que las restantes cosas tienen que ser a partir de él. Los contrarios reúnen este requisito, ya que no provienen de otra cosa, ni tampoco unos de otros. Por tal motivo, los que postularon los contrarios como principios lo hicieron acertadamente (Judson, 2018). Este punto de confluencia entre él y los predecesores se hace manifiesto sobre el cierre de I 5 (188b26-30), en donde establece que, llevados por la verdad misma (Metafísica I 3, 984b8-11.), han estado de

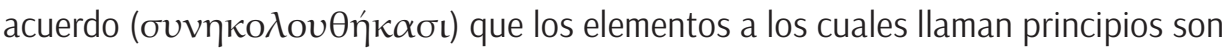
contrarios (ver Bogen, 1992, pp. 1-21).

No obstante, luego de haber concluido esta tesis, en Física I 6 Aristóteles observa que "Si no suponemos bajo los contrarios una naturaleza distinta, puede plantearse todavía otra dificultad, puesto que no vemos que los contrarios sean la sustancia de ninguna cosa". Tal como lo indica en Generación y corrupción (I 6, 322b16-19), no son los contrarios los que se modifican por acción recíproca, sino el sustrato en el cual estos inhieren. No obstante, esto trae aparejado una dificultad ulterior. Si los contrarios suponen tal sustrato, habría un principio anterior a los principios, lo cual, debido a la definición de principio dada más arriba, sería imposible (Física I 6, 189a29 ss.). Por tal motivo, se debe establecer de qué naturaleza es este sustrato y en qué medida es un tercer principio, sin entrar en contradicción con la postulación de los otros dos. Como consecuencia de esto, se debe proseguir en la indagación tomando como punto de partida los logros obtenidos hasta el momento y los problemas que se han encontrado.

Si bien esto se podrá dar por probado tras realizar el análisis del capítulo, creemos que Física I 7 está inserto en el debate que Aristóteles entabla con sus predecesores

8 Nos referimos a la clasificación entre: 1) aquellos que sostenían un principio; 2) los que formularon infinitos principios y 3) aquellos que creían en la existencia de un número limitados de principios. 
y es una continuación de la indagación que se inicia en I 2 (Berti, 2004). I 7 puede interpretarse como el momento de euporía en el proceso diaporemático ${ }^{9}$ que comienza al establecerse la necesidad de buscar los primeros principios de la física. ${ }^{10}$ Las concepciones que Aristóteles retoma en los capítulos 1-6 son su punto de partida y en algunos casos los esquemas conceptuales sobre la base de los cuales formula estos principios. En este sentido, sostenemos que su concepción de los principios surge del análisis de las tesis que considera acertadas en I 5, según las cuales 1) hay un número limitado de principios y 2) los contrarios son principios. Ahora bien, debido a que aún no se ha llegado a establecer de qué naturaleza son los contrarios y si los principios son dos o tres, la problemática aún no puede darse por concluida y la indagación debe seguir su curso.

Según Kelsey (2008), la razón de esto último se debe a que en I 6 Aristóteles se enfrenta a una aparente contradicción. Al aceptar en I 5 que hay un número limitado de principios y que los contrarios son principios, pues son anteriores y primeros, Aristóteles entra en contradicción con la siguiente tesis: un contrario no se trasmuta en su opuesto, razón por la cual debe haber un sustrato que, pese a no tener contrarios, sea capaz de recibirlos. La contradicción podría plantearse en estos términos: si debe haber un sustrato capaz de recibir los contrarios, este debe ser anterior, razón por la cual habría un principio anterior a ellos. ${ }^{11}$ Ahora bien, en virtud de la definición de principio dada en 15 , esto no sería posible. Aristóteles es consciente de que el sustrato no puede ser el único principio (Kelsey, 2008), pues esto implica aceptar la tesis monista que rechazó en 2-3, cuya consecuencia es la imposibilidad de la física. Pero su compromiso ontológico con la noción de sustrato no le permite decir que solo los contrarios son principios.

En respuesta a la problemática planteada por Kelsey, se debe indicar que es significativo que, tras formular esta contradicción, Aristóteles señale que aquello capaz de recibir los contrarios es la sustancia, pues lo que está equiparando con el sustrato es la ousía, no la materia, como en I 7. Por otra parte, es importante notar que en Categorías

9 Couloubaritsis (1980) señala que la presentación de la aporía, la diaporía y euporía constituyen los momentos que toda indagación de los principios presupone. El proceso que este modo de actuar presupone es aquel que va de "lo más conocido para nosotros" a "lo más conocido en sí".

10 Giardina (2002) sostiene que el análisis aristotélico de este capítulo es el punto culminante y el más delicado en la discusión de la noción de devenir (gígnesthai/ génesis). Tal análisis parte de la consideración del lenguaje, pero está ligado al ser. Esto se desprende, a su entender, del hecho de que está estructurado sobre la base del uso del verbo gígnomai, cuya traducción es en sí misma problemática. Por tal motivo, Berti (2004, p. 369) dirá que con esto Aristóteles imposta el problema de los principios, esencialmente como el problema de las condiciones de inteligibilidad del devenir.

11 Bostock (1982) señala que en 15 Aristóteles hace uso de la noción de prioridad de una manera que sería difícil de justificar. Según este autor, a la única conclusión a la que se puede llegar en 16 es que los principios deben incluir de alguna manera la sustancia. Ahora bien, tampoco se podría inferir esto, si se considerara que los principios solo son los opuestos. Bostock señala que no está muy claro qué sucede con este argumento cuando generalizamos la noción de un par de opuestos a la de una forma y su privación. Para este autor (1988), ni el capítulo 5 ni el capítulo 6 son completamente aporético, ya que en cada uno de ellos Aristóteles logra establecer algo: que los principios deben incluir contrarios, y que incluyen solo un par de contrarios. 
$5,^{12}$ al señalar que la ousía es capaz de recibir los contrarios, Aristóteles está pensando en los atributos accidentales. Sócrates puede ser blanco en algún momento y negro en otro. Si analizamos el tipo de contrarios que, desde la perspectiva aristotélica, son principios para sus predecesores (Física I 5) veremos que son de este tipo: a Parménides le adjudica la postulación del calor y del frío (llamados también fuego y tierra); a otros de lo raro y de lo denso y a Demócrito de lo lleno y de lo vacío (interpretados como ser y no ser respectivamente). Finalmente, al explicar cómo debe ser la generación a partir de los contrarios en I 5 los ejemplos son propiedades accidentales, tales como blanco y músico. Quizá lo que le resulta problemático no es tanto que los contrarios y el sustrato sean simultáneamente principios, sino la naturaleza de los contrarios, postulados por sus antecesores como principios. De hecho, al hablar en Física I 7 de la generación en sentido propio y establecer la necesidad de algo que permanezca, no ve problemático la coexistencia de ambas tesis: 1) necesidad del sustrato y 2) postulación de los contrarios - privación y forma-como principios, sino que la sostiene. Sin embargo, cree haber resuelto la pregunta sobre si los principios son dos o tres. Por estas razones, nos apartamos en este punto de la interpretación de Kelsey (2008) quien considera que en Física I 7 Aristóteles pretende resolver la aporía planteada en I 6, entendiendo por esto la contradicción arriba señalada.

En contraposición a la postura de Kelsey, creemos que la formulación de la aporía ${ }^{13}$ es funcional a la estrategia argumentativa implementada por Aristóteles. Si bien este autor no está comentando ni analizando este texto, sino Metafísica III, nos resulta tentadora la tesis de Aguirre Santos (2001) quien sostiene que al formular las aporías en este último escrito, Aristóteles ya tenía un andamiaje conceptual que permite resolverlas. Lo mismo podríamos decir en el caso de la Física. En Categorías 5, obra de datación temprana, y en Física I 2, 185a26-32; II 1, 192b33-34, aparece la concepción de ousía como sustrato de inherencia, que sirve de presupuesto de muchos de los argumentos expuestos en Física I 6 y 1 7. ${ }^{14}$ Paralelamente, en Metafísica II 2, escrito del mismo período que Física I y II, se sistematizan los cuatro sentidos de causas, estableciendo el rol causal que tiene la

12 En función de que en muchos de sus escritos Aristóteles remite a textos de su autoría como apoyo para defender sus argumentos, creemos que la referencia a otras obras aquí realizada es legítima, pues responde al modus operandi que Aristóteles implementa. Ejemplos de ello es la remisión que en Metafísica I 3 realiza de Física II 3 y la mención de Sobre la filosofía que aparece en Física II 2.

13 Judson (2018) no cree que la tesis de que los opuestos son principios se contradiga con la postulación de otro principio, razón por la cual, si bien no está comentando I 6, es esperable que, al formularse la necesidad de un sustrato en este último capítulo, esta autora tampoco considere que Aristóteles esté exponiendo un problema real. Code (2018) sostiene que en I 6 Aristóteles no se plantea como problema que los contrarios sea principios, sino la necesidad de que haya otro principio: el sustrato, que no puede identificarse con ninguno de los dos contrarios, razón por la cual debe ser un tercer principio. Por esta razón, para este autor, el problema que intenta resolver Aristóteles es cuál es el número de principios. Code (2018) tampoco ve una incompatibilidad entre sostener que los contrarios son principios y que es necesario la existencia del sustrato. Tiende a creer que la postulación del sustrato es una consecuencia necesaria de postular los contrarios como principios.

14 Para un análisis de la prioridad de la noción de sustancia en Aristóteles véase Mezquita (2016) 
materia como sustrato de inherencia y la forma como configuración conceptual de las entidades sensibles. Con esto queremos decir que además de las tesis que retoma de los predecesores, Aristóteles tiene presupuestos metafísicos propios que le permiten resolver la cuestión sobre los principios, sin caer en la supuesta aporía que presenta en I 6. Dado esto, se puede pensar que la formulación de la contradicción no es la presentación de verdaderos problemas, sino parte del método dialéctico implementado. El uso de este recurso se debe a que no puede haber demostración de los primeros principios. Si la hubiese, no serían principios. Por tal motivo, para dar cuenta de ellos Aristóteles procede dialécticamente (Tópicos I 2, 101b1-4). ${ }^{15}$

\section{Generación y primeros principios en Física I 7}

Al arribar a I 7, Aristóteles ha demostrado que los principios de la física deben ser más de uno, pero que su número no puede ser ilimitado (I 6, 189a11-17). Paralelamente, ha establecido que los contrarios deben ser principios (I 5, 189a9-10). Pese a esto, tal como lo vimos en el apartado anterior, la necesidad de postular un sustrato pone en evidencia que la pregunta acerca del número y naturaleza de los principios no se ha respondido aún correctamente, razón por la cual la indagación no puede cerrarse.

De la lectura de I 7, se infiere que, si la generación es el proceso por el cual algo llega a ser y los principios de la física son aquello que permite dar cuenta de este proceso, una manera posible de determinar el número y naturaleza de esos principios es partir del examen de dicha noción. Por tal motivo, en I 7, con el objetivo de resolver los problemas dejados abiertos en I 6, Aristóteles toma como punto de partida el análisis del concepto de generación. En torno a él, teje un conjunto de distinciones por medio del cual delimita el número y naturaleza de sus principios: forma, materia y privación. Pese a ello y en función de que generación se dice de muchas maneras (Física I 7, 190a31), iniciará su investigación a partir de la acepción general del término (ver Bostock, 1982). En este contexto, sostiene:

Cuando decimos que algo llega a ser otro ( $\gamma$ í $\gamma v \varepsilon \sigma \theta \alpha \iota)$ a partir de otra cosa

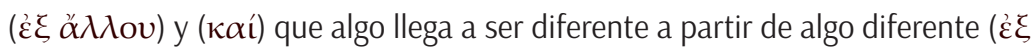

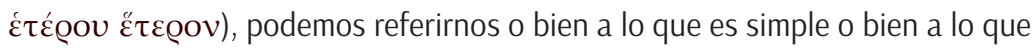
es compuesto" (Física I 7, 189b 32-34. Trad. propia).

En relación con este pasaje, lo primero que debemos subrayar son los sentidos que tiene el verbo empleado para referirse a la generación: gígnomai, ya que puede tener, al menos, dos significados: 1) nacer o "llegar al ser" y 2) devenir algo diferente, es

15 Para Code (2018), la aporía presentada en I 6 es el reto que sirve de punto de transición a su propia concepción sobre los principios. 
decir, "convertirse en tal cosa". En el primer caso, tiene un sentido ontológico y alude al pasaje del no ser al ser. El segundo, implica la transición de un modo de ser a otro. ${ }^{16}$

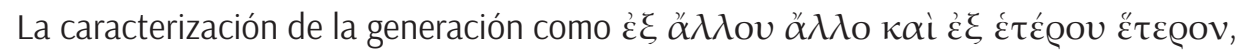
parecen reforzar la apelación implícita a este doble sentido del término. No obstante, se debe advertir que realizar esta última afirmación es más problemático. ${ }^{17}$ Esto se debe a

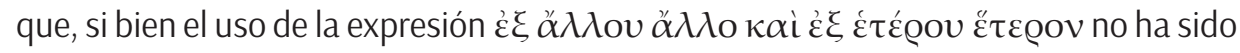
objeto de controversia, las diferentes traducciones consultadas dan cuenta de que ha sido interpretada de diversos modos, sobre todo en lo que respecta al uso del kaí. Boeri (Aristóteles, 1993) le da a kaí un sentido explicativo: "es decir". Traducido así, "llegar a ser a partir de otra cosa" sería equivalente a sostener que algo "llega a ser diferente a partir de algo diferente". Radice (Aristóteles, 2011), Stevens (1999) y Zanatta (Aristóteles, 2013) traducen kaîn como "y" (e), reproduciendo la ambigüedad que el conector tiene tanto en griego como en las lenguas modernas, esto es, permitiendo la lectura explicativa que realiza Boeri, pero también habilitando la posibilidad de entender el kaí como una conjunción ${ }^{19}$ que conecta expresiones que están en un mismo nivel sintáctico, pero que no son equivalentes. Como consecuencia de esto, esta traducción permite también

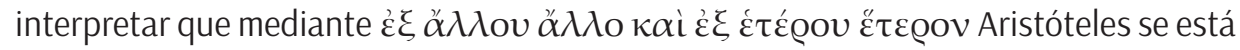
refiriendo a dos modos diferentes de entender la generación, explotando de este modo la ambigüedad semántica que el verbo gígnomai posee.

Creemos que esta última línea de lectura es más viable. Por tal motivo, suponemos que, al definir la generación como "llega a ser otro a partir de otra cosa y llega a ser diferente a partir de algo diferente", Aristóteles estaría anticipando la distinción que posteriormente trazará entre la generación de la sustancia y la transformación que esta experimenta. ${ }^{20} \mathrm{Al}$ hacer esto, retoma y corrige la concepción de los predecesores desarrollada en I 5, quienes sostienen que la generación es la transformación de algo en su opuesto. Decimos que la retoma y la corrige, pues para Aristóteles esta concepción adolece de dos problemas. El primero, formulado en I 6, radica en creer

16 Para el análisis de la polisemia de gígnomai, véase Giardina (2002; 2008).

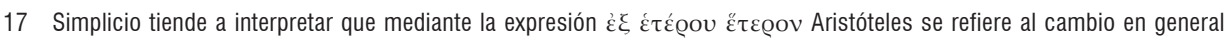
(Comentario a la Física 209 1).

Para Giardina (2002), las expresiones "alguna cosa deviene algo" y "de alguna cosa deviene algo" son dos variantes de la misma estructura del devenir en el cual dos términos contrarios entre sí, que actúan de predicados de un sujeto, se alternan entre sí.

18 Echandía (Aristóeles, 1995) y Charlton (2008) traducen kai al castellano y al inglés como si fuera una disyunción inclusiva: o y or, respectivamente.

19 Barnes traduce We say that 'one thing comes to be from another thing, and something from something different, in the case both of simple and of complex things.

20 Coincidimos con Boeri (Aristóteles, 1993) y Ross (1936), que en este pasaje ya está presente la distinción entre la generación

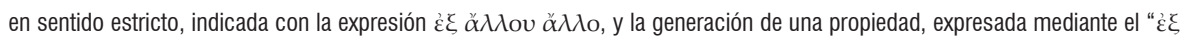

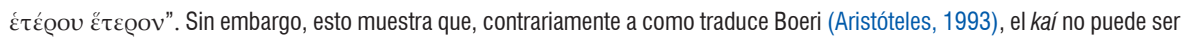
entendido en sentido explicativo. Traducirlo de este modo implica asumir que ambas construcciones son equivalentes, lo cual contradice su interpretación del texto (Aristóteles, 1993). 
que un contrario se transmuta en su opuesto, cuando en realidad este algo que se transforma no puede identificarse con ninguno de los opuestos. El segundo, tal como lo veremos más adelante, consiste en confundir, dos procesos que, según Aristóteles, son diferentes: generación y alteración.

Desde nuestra perspectiva, la formulación de los tres principios de la física surge del examen de esta resignificación de la noción de generación y de las implicancias que se desprenden de ella, al analizar cada una de las expresiones empleadas para definirla.

Para realizar esto, Aristóteles parte de la descripción de la generación de los llamados "simples". "Si bien todo lo que llega a ser es siempre compuesto"21 (Física I 7, 190b10), ${ }^{22}$ la generación puede hacer referencia a los simples, entendiendo por esto cualquier sujeto o predicado de un sujeto que no se enuncie en combinación con otro sujeto o predicado (Categorías 2 1a16-1b9), y a lo compuesto, es decir, a la combinación de los "simples" en la predicación (hombre deviene músico o no músico deviene músico). ${ }^{23}$ Los ejemplos propuestos para los simples, "hombre" y "músico", son relevantes por un motivo central: no solo permiten ilustrar los diferentes tipos de cambios aludidos mediante la

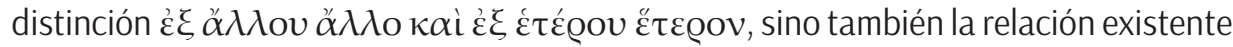
entre ellos, pues el primero, hombre, es un ejemplo de entidad sensible, mientras el segundo, músico, lo es de una cualidad.

La contraposición entre ambos tipos de generación, ejemplificados mediante "hombre" y "músico", es la base para trazar la diferenciación entre "lo que llega a ser" y "lo que llega a ser de algo" (I 7, 190a5-7). "Llegar a ser" claramente alude a la génesis como nacimiento. Si bien en este caso, como veremos más adelante, también involucrará un "llegar a ser a partir de algo", esta última acepción de la generación no necesariamente implica la gestación de una cosa, sino que puede hacer referencia lisa y llanamente a la transformación de algo, motivo por el cual el ejemplo que da Aristóteles es: "ser músico a partir de no músico". Esta última diferenciación le permitirá introducir la distinción entre "lo que permanece" y "lo que no permanece", a partir de la cual concluirá que en ambos casos debe haber algo que subyace ${ }^{24}$ a través del cambio, ${ }^{25}$ esto es, debe haber un sustrato, ${ }^{26}$ identificado hacia el final del capítulo con la materia ${ }^{27}$ y uno de

21 Para un análisis de la noción de "compuesto", véase Kelsey (2008).

22 Para el análisis de esta tesis, véase Aubenque (2008). Según Aubenque, "la alteración nos advierte que las cosas que creíamos una no lo eran, puesto que comportaba la posibilidad de convertirse en otra sin dejar de ser ella misma" (Aubenque, 2008). Según Giardina (2008), Aristóteles analiza los modos en que comúnmente nos referimos al devenir.

24 Bostock (1982) subraya que hay una ambigüedad en la frase "lo que subyace" (hupokeímenon), pues mediante su uso Aristóteles no solo afirma la persistencia de algo a lo largo del cambio, sino también la transformación de un sujeto en tal o cual cosa.

25 Según Berti (2004), con el descubrimiento del sustrato, Aristóteles ha puesto en evidencia por primera vez la necesidad de que el devenir sea el devenir de un sujeto.

26 Para el análisis de la polisemia "sustrato", véase Bastit (2002). Según este autor, tanto la forma, como la materia y el compuesto pueden ser sustrato primero, pues los tres son posibles de ser sujeto de predicaciones. Por tal motivo, en unos casos el sujeto de la generación será la sustancia, pero en el caso de la generación absoluta, la materia.

27 Cabe destacar que, como lo indica Carbonell (2014), Aristóteles califica a la forma como sujeto o sustrato (Metafísica VII 3,1029a1-3). Esto se debe a que "la forma es la que da cuenta de la identidad de la sustancia, de su persistencia a lo largo del tiempo. En tal 
los principios de la física. Veamos, pues cómo llega a la postulación de este principio sobre la base del siguiente esquema:

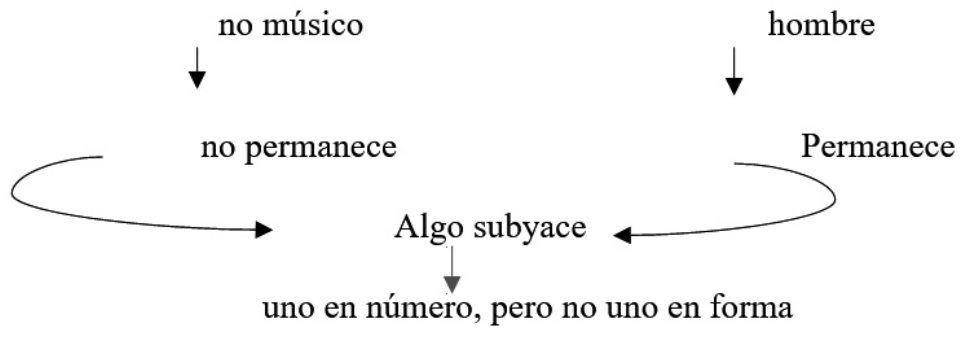

Aristóteles comenzará su análisis por lo que no permanece, razón por la cual examinará la expresión "no músico deviene músico", que en rigor presupone "hombre no músico deviene músico". ${ }^{28}$ Al igual que algo deja de estar frío cuando se calienta, o seco cuando se moja, el que no es músico o no blanco, al adquirir las propiedades contrarias, deja de poseer las que tenía antes, por tal motivo, de estas propiedades contrarias decimos que no permanecen. Ahora bien, para que este pasaje de un contrario al otro sea posible es necesario un sustrato, pues "no vemos que los contrarios sean la sustancia de ninguna

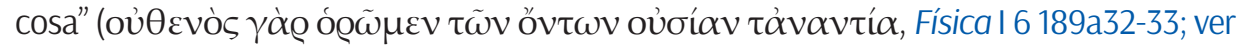
Kelsey, 2008, p. 203). Dicho sustrato debe permanecer a través del cambio o del pasaje de un contrario al otro (Categorías 5 2b1-5); por lo tanto, al aceptar esto, se debe admitir que hay algo que permanece. Es evidente que en este pasaje Aristóteles supone su concepción de la sustancia presentada en Categorías 5. Por esa obra, sabemos que la sustancia es el sujeto de inherencia de las propiedades, por tal motivo, es aquel sustrato que subyace en el pasaje de un contrario al otro (Categorías 5 3b24-25), pues, si bien carece de contrarios, es capaz de recibirlos (Categorías 54 a10 ss.). La sustancia es 1) aquello que ni se dice de un sujeto, ni está en un sujeto (Categorías 5 2a11-12) y 2) aquello respecto de lo cual se predican e inhieren las restantes categorías. En el ejemplo dado por Aristóteles lo que permanece es "hombre". Citando a Carbonell (2007) se puede decir que no es propiamente el "hombre -con-determinada- cualidad" el que llega a ser "hombre-con-otra-cualidad", sino que aquello que llega a ser -es decir, aquello que cambia-es, sin más, el hombre. En este caso, coinciden el sujeto gramatical con el sujeto del cambio. ${ }^{29}$

No obstante, hay que tener en cuenta que en este primer libro de la Física Aristóteles dice expresamente que no ha resuelto qué es la sustancia. Los dos candidatos que

sentido, la forma explica que la sustancia sea el sujeto de los accidentes" (Carbonell, 2014, p. 65) .

28 El motivo por el cual adopta este punto de partida es que procede de lo más conocido para nosotros a lo más conocido en sí. Es más intuitivo entender la generación de las propiedades de la sustancia que la de esta última. Véase Simplicio Comentario a la Física 2091 212, 19-20.

29 Severino (1995) indica esto diciendo que no es lo opuesto lo que se convierte en su opuesto, sino el substrato que recibe las determinaciones contrarias. 
presenta son la materia y la forma, pero no el compuesto de ambos (Física 17, 191a19-20). Con lo cual, cuando Aristóteles afirma "hombre permanece", es problemático establecer si se refiere a que la entidad sensible permanece a través del cambio, esto es, en la medida en que adquiere algunas propiedades accidentales y pierde otras, o si refiere a que, de una entidad sensible, la forma, lo que este individuo es esencialmente, se mantiene, mientras que el resto de sus características accidentales cambian. Creemos que, en tanto que Aristóteles está realizando un análisis de la estructura de la predicación ${ }^{30}$ pero que claramente reviste un carácter ontológico, es muy probable que se esté refiriendo al sujeto gramatical hombre, ${ }^{31}$ teniendo en cuenta que por medio de "hombre" se está haciendo referencia a un compuesto de materia y forma, el cual está determinado y se define esencialmente por esta última. ${ }^{32}$ Con esto queremos indicar que Aristóteles está explotando la complejidad que la noción de ousía tiene, ya que una entidad sensible es para Aristóteles sustancia (Categorías 5), pero, desde una perspectiva causal, su forma, aquello que hace que sea lo que es, es ousía en sentido prioritario. La forma, por otra parte, tiene una particularidad que la entidad sensible (compuesto de materia y forma) no posee, es eterna (Metafísica VII 8, 1033b5-10). Por lo tanto, de ella se puede decir que siempre permanece, ya que no está expuesta a la generación y corrupción. Ahora bien, por esta misma razón, cuando se refiere a la generación de la ousía en Física l 7, 190a31-190b5 la forma no puede ser el referente de esto último, sino la entidad sensible, respecto de la cual, materia y forma son principios.

En este último caso, pese a que "no músico deviene músico", presupone un individuo que permanece, en tanto este individuo es una entidad sensible, se debe aceptar que también está sujeto a generación y corrupción. Dado esto, se puede decir que en un sentido permanece, pero en otro no. Decimos que hombre permanece cuando nos referimos a él como el sujeto respecto del cual realizamos predicaciones y cuando es pensado en relación con las alteraciones que, en tanto sustancia sensible, es capaz de experimentar. Sin embargo, estamos obligados a afirmar que no permanece cada vez que nos referimos a él como una entidad sujeta al devenir. No obstante, aun en este caso, también es necesario que algo permanezca. Esto se debe a que Aristóteles le concede a los eléatas que no es posible la generación del no ser absoluto entendiendo

30 Con Severino (1995) podemos decir que "la intención de Aristóteles es mostrar cómo el lenguaje expresa la estructura del devenir, y que, por lo tanto, cómo la reflexión conceptual hace explícito lo que está implícito en la inmediatez de la palabra” (p. 56). Tal como lo indica Mié (2006), "Ia física se delimita allí, como una tarea de reflexión sobre los presupuestos que introducimos en nuestras creencias más relevantes y destacadas, y en nuestras expresiones lingüísticas acerca de las cosas sujetas a movimiento" (p. 71). Esta modalidad de análisis es una forma de ir de "Io más conocido para nosotros" a "lo más conocido en sí". El uso del verbo "decimos" en Física I 7, 189b 32-34, así como el análisis de los modos de referirnos a la generación presente a lo largo de todo este capítulo permite corroborar esta línea interpretativa.

31 Como indica Berti (2004), el leguaje permite distinguir las cosas que en la realidad están en unidad, esto es, permite diferenciar la forma, o sea la esencia, del resto de las cualidades que son una en número.

32 Couloubaritsis (1980) sostiene que Aristóteles toma como premisa de su análisis su concepción de la pluralidad del ser y la afirmación de que la esencia es el sujeto de toda predicación. 
por esto la completa ausencia de algo (ver Física I 8, 191b13-14; Física V 1, 225a20-26). Según Aristóteles, "si 'en sentido absoluto (ő $\lambda \omega \varsigma)$ ' significa llanamente el no ente ( $\mu$ ทे òv), esto equivaldrá a una negación universal de todas las cosas y, en consecuencia,

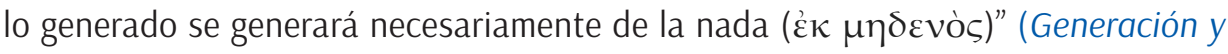
corrupción I 3, 318a11-13). Para poder dar cuenta de la generación como un tránsito del no ser al ser sin entrar en contradicción con la premisa aceptada a los eléatas, Aristóteles debe resignificar el sentido de estas dos nociones -las de ser y no ser-. Dado esto, debe admitir que "de algún modo hay un llegar a ser de lo que no es (غ̇ં

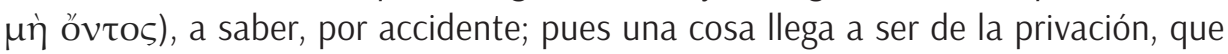

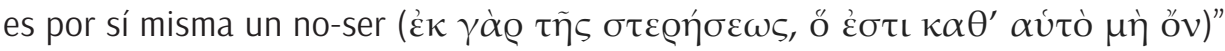
(Física I 8, 191b14-16. Trad. Echandía, modificada; ver Física I 9, 192a3-6). Para no caer en la necesidad de postular un no ser existente o determinaciones que existan separadas de "un esto", Aristóteles establecerá la existencia de un sustrato no determinado a partir del cual las entidades sensibles se generan. De aquí que postula la materia ${ }^{33}$ (Generación y corrupción I 3, 318a1-2) como principio de la generación. Esto supone que las cosas están compuestas de un sustrato en el cual inhieren tanto sus propiedades esenciales, que lo determinan como lo que es, como las accidentales. Cuando la cosa se degenera y se destruye como tal, este sustrato permanece, aunque en un estado de indeterminación. Dicho sustrato es susceptible de ser informado nuevamente, de modo tal de dar paso a la generación de una nueva cosa (Acerca de la generación y corrupción I 3-4).

Sobre la base de lo aquí argumentado, se puede decir que, si bien "llegar a ser a partir de", se dice principalmente de lo que no permanece (músico), también se predica de lo que permanece, pues es lícito afirmar "la estatua llega a ser a partir del bronce" indicando de este modo la necesidad del sustrato. No obstante, no se puede decir que el bronce llegue a ser estatua. Esto quiere decir que no se puede explicar la generación de las entidades sensibles en los mismos términos en que se explica la adquisición de una cualidad. Como consecuencia de esto, Aristóteles continuará su análisis volviendo sobre su definición de génesis:

'Llegar a ser' ( $\gamma \hat{\prime} \gamma v \varepsilon \sigma \theta \alpha \iota)$ se dice en muchos sentidos: en algunos casos no se

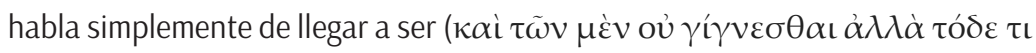
$\left.\gamma^{\prime} \gamma \nu \varepsilon \sigma \theta \alpha \iota\right)$, sino de llegar a ser algo particular, pero sólo de las sustancias se dice que llegan a ser en sentido absoluto ( $\dot{\alpha} \pi \lambda \tilde{\omega} \varsigma \delta \dot{\varepsilon} \gamma \dot{\gamma} \gamma v \varepsilon \sigma \theta \alpha \iota \tau \tilde{\omega} v$ oủ $\sigma \tilde{\omega} v$ uóvov) (Física 190 a 31-33). Cuando no se trata de las otras cosas, es evidente

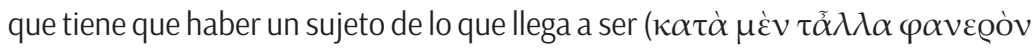

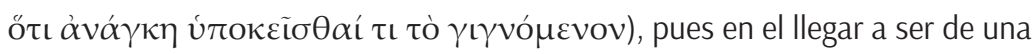

33 Giardina (2008) dirá que la permanencia del sustrato resuelve la dificultad eleática de la generación del no ser. No obstante, como lo indica Mié (2006), "para ser sustrato del cambio la materia debe mantener una relación constitutiva con el producto" (p. 77). 
cantidad o una cualidad o una relación o un donde hay siempre un sujeto de ese llegar a ser, ya que solo la sustancia no se predica de ningún otro sujeto, mientras que todo lo demás se predica de la sustancia. Pero que también las sustancias, y

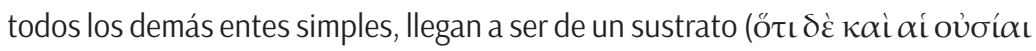

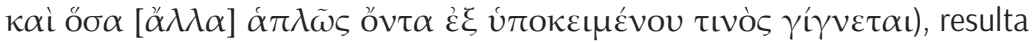
evidente si se lo examina con atención. Porque siempre hay algo subyacente de lo que procede lo que llega a ser; por ejemplo, los animales y las plantas proceden de la semilla (Física I 7, 190a31-190b5. Trad. Echandía, modificada).

En este pasaje, Aristóteles explicita los dos tipos de generación que estaban prefigurados en la acepción general con la que abrió el capítulo. El primero, el vinculado con llegar a ser esto, es el que describimos al hablar de aquello que no permanece, esto es, cuando nos referimos a la adquisición de una cualidad, cantidad, etc., que puede experimentar una entidad sensible. En relación con esto, en este pasaje, Aristóteles explicita el supuesto que estaba operando como telón de fondo en los pasajes precedentes, a saber, la primacía de la ousía por sobre el resto de las categorías. Sin embargo, en este caso, podemos hablar de generación solo en un sentido relativo. Esto se debe a que la generación en sentido propio no es cualquier tipo de cambio o proceso de devenir, sino solo aquel referido al surgimiento de las entidades sensibles. En Acerca de la generación y corrupción sostendrá:

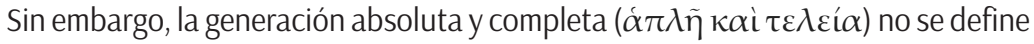

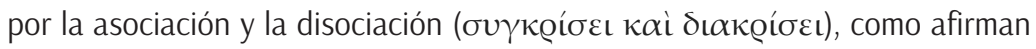
ciertos pensadores que consideran que la alteración consiste en un cambio en lo

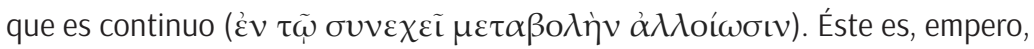
el punto en el cual todas estas doctrinas yerran. En efecto, la generación y la corrupción absolutas no se producen por asociación y disociación, sino cuando

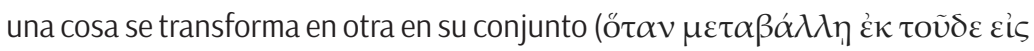

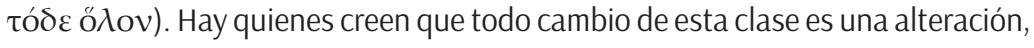
pero, sin embargo, hay diferencia. En el sujeto del cambio están presentes lo correspondiente a la definición esencial y lo correspondiente a la materia ('Ev

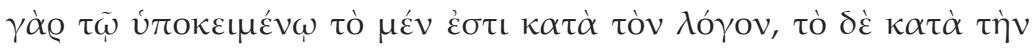

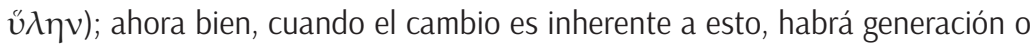

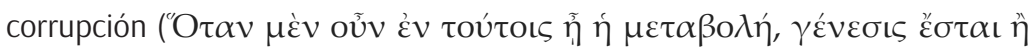
$\varphi \theta$ o@ó), mientras que, cuando es inherente a las afecciones y es accidental,

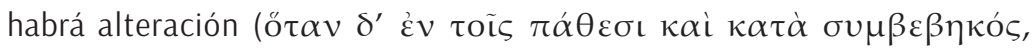

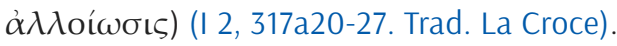

La lectura de los pasajes de la Física y Acerca de la generación y corrupción nos permite trazar las siguientes comparaciones entre los dos tipos de génesis: aquel que podríamos llamar "generación en sentido absoluto" y aquel otro que podríamos calificar de "generación en sentido relativo": 
Generación en sentido propio: solo se dice en relación con la ousía (Física I 7, 190a32-33)

Llegar a ser en el sentido de generarse

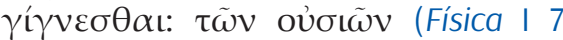
190a32)

En Acerca de la generación y corrupción

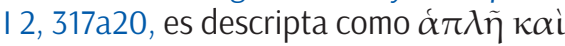
$\tau \varepsilon \lambda \varepsilon i ́ \alpha$

Supone un sustrato a partir del cual llega a ser (Física I 7, 190b1-2): la materia

Cambio total de una cosa en otra cosa: cambio inherente al lógos y materia (Generación y corrupción I 2, 317a26-27)
Generación en sentido relativo. Se dice en relación con las restantes categorías

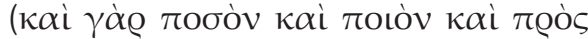

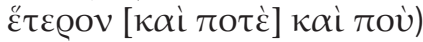

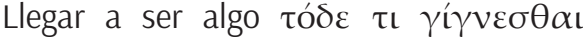
(Física | 7, 190a32)

Supone un sustrato a partir del cual llegan a ser: la ousía, pues las cosas generadas de esta forma se dicen de la sustancia y están en una sustancia (Física I 7, 190a33-36)

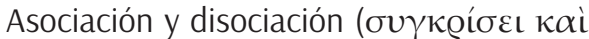

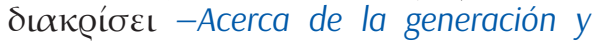
corrupción I 2, 317a21). Cambio inherente a las afecciones de una entidad sensible compuesta de materia (húle) y forma (eîdos /lógos) (Generación y corrupción I 2, 317a24-25).

Desde la perspectiva aristotélica, el problema de los predecesores que postularon los contrarios como principios (Física I 5) es que confunden la alteración con la generación, razón por la cual dan cuenta del primer proceso y no del segundo. Por este motivo, no establecieron correctamente el número y la naturaleza de los principios de la física. Para Aristóteles, si bien alteración y generación son formas de cambios, no implican el mismo tipo de cambio. El primero es un cambio de las afecciones de la sustancia, entiendo por esta a la entidad sensible (algo llega a ser algo), mientras que el segundo alude al surgimiento de la sustancia propiamente dicha (llegar a ser) (Acerca de la generación y corrupción I 2, 317a26-27). Para entender esta diferencia, creemos que se debe tener en cuenta que, según Aristóteles, "la palabra 'metabole’34 indica algo 'después de' otro

34 Waterlow (1982), sostiene que metabolé tiene el sentido más general de cambio. En algunos pasajes (200b, 32-33; 201a2, 8-9), su uso es intercambiable con kínesis. Sin embargo, en V 1, Aristóteles distingue ambos términos, restringiendo kínesis al cambio no sustancial. Para esta autora, mientras metabolé es un cambio de un estado a otro, kínesis es un proceso. Para la relación génesis - metabolé véase Couloubaritsis (1980). Según este último autor, mientras en I 1, en el contexto de la crítica a los eléatas, metabolé tiene el sentido ordinario de modificación completa de una cosa, en I 7 "significa una modificación acompañada de la presencia o ausencia de uno de los contrarios" (Couloubaritsis, 1980, p. 52) y en el capítulo 8 refiere al cambio en general. En estos dos últimos casos, advierte este autor, el uso del término es completamente marginal y no afecta al argumento en torno del devenir. Desde su perspectiva, no es casual que Aristóteles evite el uso de metabolé en el contexto de la discusión del devenir. Couloubaritsis subraya que devenir, cambio y movimiento no son términos sinónimos (Couloubaritsis, 1980). Las lecturas más canónicas, dentro de las cuales incluye la de Simplicio, tienden a considerar que metabolé es un tipo 


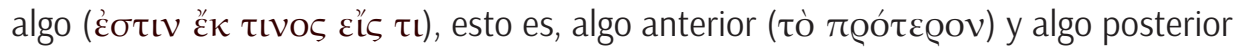

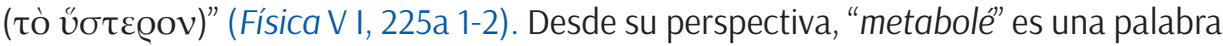
polivalente, ya que mediante ella podemos referirnos al cambio: 1) que va de un sujeto a otro sujeto; 2) el que implica un pasaje desde un sujeto a un no sujeto y 3) el que se produce entre un no sujeto y un sujeto. El primer tipo de cambio es el cualitativo, el cuantitativo y el locativo (Física V 1, 225b5-9). Implica un pasaje de un contrario al otro. "En este caso, la expresión 'ser no blanco' no indica la negación de 'ser blanco', sino la privación" (Berti, 2004, p 313). Este tipo de movimiento es calificado también como kínesis (ver Física V 1, 225a1-6; Berti, 2004, pp. 312-313). Los dos últimos tipos de cambios -el que va de un sujeto a un no sujeto y el que va de un no sujeto a un sujeto-, se identifican con la generación y corrupción, respectivamente. Tal cambio no

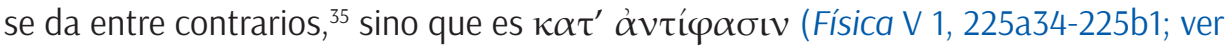
Metafísica X 4, $1055 a 3$ ss.), ${ }^{36}$ por eso no puede ser un movimiento.

Esta clasificación nos sirve para advertir que, si bien la generación es un tipo de cambio, no sucede lo mismo en el sentido inverso: no todo cambio es una generación. Es sobre la base de esta definición de generación que Aristóteles termina por explicitar cuáles son los tres principios de la física: materia, forma y privación.

Según él, la generación en sentido absoluto es la determinación en acto de una materia que preexiste (Física I 9, 192a30-31). Ahora bien, si tomamos como punto de partida esta definición, debemos advertir que, junto con la materia, es necesario postular como principio a la forma. La generación, en este caso, será un cambio inherente a lógos ${ }^{37}$ y húle porque se trata del proceso arriba descrito por el cual la materia indeterminada pasa de la privación de la forma o de la indeterminación a ser un esto determinado.38 La generación es el proceso por el cual la materia deviene una entidad sensible debido a que adquiere su forma, su esencia o su "qué es ser". ${ }^{39}$ Por tal motivo en

de cambio que engloba a la generación y el movimiento. Metabolé sería un género de cambio, en relación con el cual los dos últimos serían sus respectivas especies. Sin embargo, para Couloubaritsis (1980), en la medida en que la plurivocidad del ser se corresponde con una plurivocidad de cambios, al igual que en el caso del ser, se torna imposible hablar del cambio como un género, razón por la cual la interpretación arriba sintetizada, sería inviable.

35 Según Bostock (1982) ninguno de los opuestos es simplemente la negación del otro. Sin embargo, forma y privación se niegan recíprocamente, razón por la cual, para este autor, son conceptos mucho más generales que el de opuesto. El motivo por el cual este autor subraya esto es que en I 5 , a diferencia de I 7, Aristóteles sostiene la tesis de que el cambio siempre es entre opuestos (Física I 5, 188a31 ss.).

36 Para el análisis de los contrarios en Aristóteles véase Bogen (1992).

37 El uso de lógos en el pasaje de Acerca de la generación y corrupción es significativo, pues pareciera ser equivalente a eîdos. En Física I 7, 190a16, realiza de forma expresa esta equiparación. Allí, al establecer que el sustrato y "aquello que se llega a ser" es uno en número, pero no en forma (eîdos), Aristóteles identifica eîdos con lógos. Si bien la traducción de lógos en estos pasajes es problemática, es claro que, si no se lo identifica con definición, lo cual creemos viable, es un principio explicativo que da cuenta de lo que la cosa es esencialmente.

38 Véase Mié (2006). Allí Mié sostendrá que la materia es un principio constitutivo no accidental en la medida en que guarda relación con la forma (Física 191a11, a9-11).

39 Quizá por esto, se puede decir que hay una preeminencia de la forma por sobre los otros dos principios: "El cambio y lo que cambia se denominan según aquella forma hacia la cual se produce algo, más que según la materia (o la privación) a partir de la cual tiene lugar 
Metafísica X 1055b 11, Aristóteles señala que la generación en la materia se produce a partir de los contrarios: o bien a partir de la posesión de la forma o a partir de la privación de la forma. Para Aristóteles:

También nosotros afirmamos que en sentido absoluto nada llega a ser de lo que no es, pero que de algún modo hay un llegar a ser de lo que no es, a saber, por accidente; pues una cosa llega a ser de la privación, que es de suyo un no-ser (Física I 8, 191b 13-16. Trad. Echandía modificada).

Tal como lo indica Aubenque (2008, p. 360), en este proceso, la forma es el atributo por adquirir. Como tal se contrapone a la materia, que es aquello en lo cual este atributo inhiere ${ }^{40}$ y a la privación, que puede ser definida como la ausencia de esta forma y que, por este motivo, solo se constituye como principio de modo retroactivo, "como carencia y expectativa de aquello a lo que el sujeto ha llegado de hecho" (Aubenque, 2008, p. 361). En este esquema, la materia es lo que preexiste y permanece a través del cambio y que puede ser considerada tanto como ente en potencia o como no ente en acto (Generación y corrupción I 3, 317b31. Ver Quevedo, 1997). Tanto la forma como la materia son principios per se; en cambio, la privación lo es por accidente (Física I 7, 190b27; ver Quevedo, 1997). Decimos que la forma y la materia son principios por sí mismos, pues intervienen como condición necesaria en toda generación. Esto se debe a que aún en el caso "no músico deviene músico", que es un ejemplo de generación relativa, la ousía es aquello que subyace y en lo cual inhiere la propiedad de ser músico. Ahora bien, pese a que en este caso, la ousía puede ser equivalente a la entidad sensible, en tanto y en cuanto esta última es un compuesto de materia y forma, presupone a estas dos últimas como principios del devenir. La entidad sensible depende ontológicamente de estos dos principios para poder existir, razón por la cual los principios de la generación terminan siendo reductibles a ellas. Esto es significativo porque, a nuestro modo de entender, es la causa por la cual dos de los tres principios de todo tipo de generación ${ }^{41}$ son materia y forma, pero no la entidad sensible.

La privación, en cambio, se define como tal en contraposición con la forma, razón por la cual se erige como principio no por sí misma, sino en tanto se opone a la forma. Dado esto, es principio por accidente.

De este modo, hacia el final del capítulo 7 Aristóteles dará por respondidos los interrogantes con los cuales inició su indagación: el número y naturaleza de los principios. La explicación de la generación a partir de estos tres principios le permite a Aristóteles explicar cómo es posible el movimiento y, por lo tanto, el objeto de la física,

el movimiento (Física. V 1, 224b7-8; 5, 229a25-27; II 1, 193b3-7; Mié, 2006, p. 73).

40 Tal como lo indica Batist (2002) la materia es la condición negativa a partir de la cual es posible la actualidad de la forma, por eso, en cierto sentido, califica de ousía.

41 Recordamos en este punto que el proyecto de Aristóteles para el presente capítulo es tratar la generación en general, con lo cual es esperable que los tres principios buscados puedan explicar cualquier proceso de devenir. 
como un cambio del no ser al ser, sin por ello caer en las aporías de los eléatas, que los forzaron a suponer un único ser, y sin suponer una infinidad de principios que harían de la ciencia una tarea imposible al tener por objeto lo indeterminado.

\section{Conclusión}

Hemos intentado demostrar que en Física I 7 debe leerse a la luz de los capítulos precedentes, en los que Aristóteles discute la naturaleza y número de los principios con sus predecesores. De las opiniones de sus antecesores, Aristóteles toma como punto de partida dos tesis: la primera es que, si bien debe haber más de un principio, su número debe ser limitado. La segunda es que los contrarios son principios. Sin embargo, en I 6 Aristóteles establece la necesidad de un sustrato, en el cual los contrarios inhieran, lo cual, dada la definición de principio de 15, sería aparentemente contradictorio. Pese a esto, Aristóteles no parece cuestionarse, cuál de los dos es principio, si los contrarios o el sustrato, sino que cree que el problema es establecer si los principios son dos o tres. En I 7, Aristóteles pretende resolver esta cuestión que permite de manera concluyente responder la pregunta que inspira todo el libro: ¿cuáles y cuántos son los principios?

En la tercera parte, analizamos las distinciones que le permiten concluir que los principios buscados son tres: materia, forma y privación. Para Aristóteles, toda generación, ya sea la generación de la sustancia (sentido absoluto) o ya sea la adquisición de una propiedad o cualidad (sentido relativo), supone llegar a ser a partir de algo. Esto implica que en ambos casos hay algo que debe permanecer. En el caso de las cosas que no permanecen (no músico, músico, blanco no, blanco, etc.), esto que subyace es la ousía, aunque Aristóteles aclara hacia el final del capítulo que no ha resuelto qué debe entenderse por sustancia: si es la forma o la materia; en el caso de la generación de las entidades sensibles es la materia. Dado que Aristóteles acepta que es imposible la generación a partir del no ser en sentido absoluto, debe sostener que, aun en el caso del surgimiento de las entidades sensibles, algo debe permanecer o subyacer. Este algo no puede ser una naturaleza determinada, como sostuvieron algunos de sus predecesores, sino la materia que carece de determinación.

En ambos casos, la generación es el proceso por el cual algo, entiéndase por esto una ousía (generación relativa) o la materia indeterminada (generación absoluta), privado de una propiedad la adquiere. En este último caso, esto es, cuando se trata de la generación de las ousíai o de las cosas que son en sentido propio (I 7, 190b 1-2), esta propiedad no es cualquier atributo, sino sus determinaciones esenciales, es decir, la forma o esencia. Cuando la materia indeterminada o privada de la forma, es informada por esta última, pasa de la privación a ser un esto determinado, completándose de esta forma el devenir de la cosa en cuestión. Por esta razón, materia, forma y privación deben ser los únicos tres principios. Sin embargo, solo la materia y la forma son principios en sentido estricto, mientras que la privación lo es accidentalmente. 


\section{Referencias}

Aguirre Santos, J. (2001). Aristóteles: Aporética como metodología. El caso de Metafísica B. Revista Laguna, 9, 37-52.

Aguirre Santos, J. (2015). Dialéctica y filosofía primera. Lectura de la Metafísica de Aristóteles. Universidad de Zaragoza.

Aristotele. (2008). Sulla generazione e la corruzione (G. I. Giardina, Introduzione, traduzione e note). ARACNE Editrice S. R. L.

Aristóteles. (1982). Tópicos. En Organon, vol. I (M. Candel Sanmartin, Trad. Introducción y notas) (pp. 89-306). Gredos.

Aristóteles. (1982). Categorías. En Organon, vol. I (M. Candel Sanmartin, Trad. Introducción y notas) (pp. 23-77). Gredos.

Aristóteles. (1983). Metafísica (T. Calvo Martínez, Trad. Introducción y notas). Gredos.

Aristóteles. (1987). Acerca de la generación y corrupción (E. La Croce, Trad. Introducción y notas). Gredos.

Aristóteles. (1993). Física (M. Boeri, Trad. Introducción y notas). Biblos.

Aristóteles. (1995). Física (G. Echandía, Trad. Introducción y notas). Gredos.

Aristóteles. (2011). Física (R. Radice, Trad. Introduzione e commento). Bompiani.

Aristóteles. (2013). Física (M. Zanatta, Trad. Introduzione e commento). Editrice Torinese.

Aristotle. (1999). Physique (A. Stevens, Trad. et commentaire). Vrin.

Aubenque, P. (2008). El problema del ser en Aristóteles (V. Peña, Trad.). Escolar y Mayor Editores. (Original publicado en 1962).

Bastit, M. (2002). Les quatre causes de lêtre selon la philosophie première d'Aristote. Éditions Peeters.

Berti, E. (2004). Aristotele: dalla dialettica alla filosofia prima. Bompiani. (Original publicado en 1977).

Berti, E. (2008). Struttura e significato della Metafisica di Aristotele. EDUSC.

Boeri, M. (2006). Aristóteles contra Parménides: el problema del cambio y la posibilidad de una ciencia física. Tópicos, 30 bis, 45-68.

Bogen, J. (1992). Change and Contrariety in Aristotle. Phronesis, 37(1), 1-21. https://doi.org/10.1163/156852892321052632

Bolton, R. (1990). The Epistemological Basis of Aristotelian Dialectic. In D. Devereux \& P. Pellegrin (Eds.), Biologie, logique et métaphysique chez Aristote (pp. 185-236). Editions Centre National de la Recherche Scientifique.

Bolton, R. (2003). Aristotle's Method in Natural Science: Physics I. In L. Judson (Ed.), Aristotle's Physics. A Collection of Essays (pp. 1-29). Clarendon Press.

Bostock, D. (1982). Aristotle on the Principles of Change in Physics. In I. M. Schofield \& M. Nussbaum (Eds.), Language and Logos (pp. 179-96). Cambridge University Press. https://doi.org/10.1017/CBO9780511550874.011 
Bruce, L. \& Robertson, L. A. (2002). Commentary on Book Alpha Elatton of Aristotle's Metaphysics. Animus, 7, 1-22.

Brunschwig, J. (1990). Remarques sur la communication de Robert Bolton. In D. Devereux, \& P. Pellegrin (Eds), Biologie, logique et métaphysique chez Aristote (pp. 237-262). Editions Centre National de la Recherche Scientifique.

Carbonell, C. (2007). Movimiento y forma en Aristóteles. Ediciones Universidad de Navarra, S. A. Carbonell, C. (2014). La forma como sujeto: ¿un desliz de Aristóteles? Eidos como sujeto y garante de la identidad. Estudios de Filosofía, 48, 49-72. https://revistas.udea.edu.co/ index.php/estudios_de_filosofia/article/view/18346

Cárdenas Mejía, L. G. (1998). ¿Dialoga Aristóteles con Parménides? Estudios de Filosofía, 17-18, 59-71. https://revistas.udea.edu.co/index.php/estudios_de_filosofia/article/view/338009

Cassin, B. (1989). Parle si tu es un homme. En B. Cassin \& M. Narcy (Eds), La décision du sens. Le livre Gamma de la Métaphysique d'Aristote (pp. 9-60). Vrin.

Charlton, W. (2008). Aristotle's Physics I- II, Introduction and commentary. Clarendon Press. (Original publicado en 1970).

Code, A. (2018). Physics I 6. En D. Quarantotto (Ed.), Aristotle' Physics Book I. A systematic Explanation (pp. 154-177). Cambridge University Press. https://doi.org/10.1017/9781108181853.008

Couloubaritsis, L. (1980). La Physique d'Aristote. L'avenement de la science Physique. Peeters.

Dancy, R. M. (1975). Sense and Contradiction. A Study in Aristotle. Reidel. https://doi.org/10.1007/978-94-010-9770-3

Díaz, M. E. (2014). Alternativas para pensar la génesis. El mapa aristotélico de los que abordaron el problema de la generación. En G. Marcos \& M. E. Díaz (Eds.), El filósofo y sus adversarios en los escritos de Platón y Aristóteles (pp. 135-151). Rhesis.

Giardina, G. I. (2002). Fondamenti della Fisica. Analisi critica di Aristotele, Phys. I. Symbolon. Irwin, T. (1988). Aristotle's Principles. Clarendon Press.

Judson, D. (2018). Physics I 5. En D. Quarantotto (Ed.), Aristotle' Physics Book I. A systematic Explanation (pp. 130-153). Cambridge University Press. https://doi.org/10.1017/9781108181853.007

Kelsey, S. (2008). The Place of I 7 in the Argument of Physics I. Phronesis, 53, 180-208. https://doi.org/10.1163/156852808X278721

Mesquita, A. P. (2016). Platón y Aristóteles. Dos ontologías en confrontación. Estudios de Filosofía, 53, 57-79. https://doi.org/10.17533/udea.ef.n53a04

Mié, F. (2006). Persistencia y continuidad del sustrato material en la Física de Aristóteles. Tópicos, 30 bis, 69-100.

Mié, F. (2009). Dialéctica y ciencia en Aristóteles. Signos Filosóficos, 11(21), 9-42.

Quevedo, A. (1997). Aristóteles en torno a la privación y la causalidad. Anuario Filosófico, 30, 71-99. 
Ross, W. D. (1936). Aristotle's Physics. Introduction and commentary. Clarendon Press. https://doi.org/10.1093/actrade/9780198141099.book.1

Rossi, G. (2001). Algunas notas sobre la discusión con los eléatas en Física I de Aristóteles. Tópicos, 20, 13 -159.

Rossi, G. (2017). Going through Aporiai: The Critical Use of Aristotle's Dialectic. Oxford Studies, Ancient Philosophy, 52, 209-256. https://doi.org/10.1093/oso/9780198805762.003.0006

Severino, E. (1995). I principi del divenire. Libro primo della Fisica. La scuola.

Simplicius. (1882). Commentaria in Aristotelem Graeca: in Physika (H. Diels, Ed.). De Gruyter.

Spangenberg, P. (2014). La figura de Protágoras en la prueba aristotélica contra el negador del principio de no-contradicción. En G. Marcos \& M. E. Díaz (Eds.), El filósofo y sus adversarios en los escritos de Platón y Aristóteles (pp. 153-177). Rhesis.

Vigo, A. (2016). Filosofía y dialéctica en Aristóteles: un enfoque sinóptico. Hypnos, 36(1), 1-24.

Waterlow, S. (1982). Nature, Change, and Agency in Aristotle's Physics: A philosophical study. Clarendon Press. https://doi.org/10.1093/acprof:0so/9780198246534.001.0001

Wright, M. R. (1981). Empedocles. The Extant Fragments. Introduction, commentary and concordance. Yale University Press. 